\title{
SYMMETRY ANALYSIS OF THE KLEIN-GORDON EQUATION IN BIANCHI I SPACETIMES
}

\author{
A. PALIATHANASIS \\ Dipartimento di Fisica, Universita' di Napoli, "Federico II", Compl. Univ. di Monte S. Angelo, \\ Via Cinthia, I-80126, Napoli, Italy \\ INFN Sez. di Napoli, Compl. Univ. di Monte S. Angelo, Via Cinthia, I-80126, Napoli, Italy \\ paliathanasis@na.infn.it \\ M. TSAMPARLIS \\ Faculty of Physics, Department of Astrophysics - Astronomy - Mechanics, University of Athens, \\ Panepistemiopolis, Athens 157 83, Greece \\ mtsampa@phys.uoa.gr \\ M. T. MUSTAFA \\ Department of Mathematics, Statistics and Physics, College of Arts and Sciences, Qatar \\ University, Doha 2713, Qatar \\ tahir.mustafa@qu.edu.qa
}

\begin{abstract}
In this work we perform the symmetry classification of the Klein Gordon equation in Bianchi I spacetime. We apply a geometric method which relates the Lie symmetries of the Klein Gordon equation with the conformal algebra of the underlying geometry. Furthermore, we prove that the Lie symmetries which follow from the conformal algebra are also and Noether symmetries for the Klein Gordon equation. We use these resutls in order to determine all the potentials in which the Klein Gordon admits Lie and Noether symmetries. Due to the large number of cases and for easy reference the results are presented in the form of tables. For some of the potentials we use the Lie admitted symmetries to determine the corresponding invariant solution of the Klein Gordon equation. Finally, we show that the results also solve the problem of classification of Lie/Noether point symmetries of the wave equation in Bianchi I spacetime and can be used for the determination of invariant solutions of the wave equation.
\end{abstract}

Received (Day Month Year)

Revised (Day Month Year)

\section{Introduction}

The study of differential equations in a general Riemannian space involves investigation of equations containing arbitrary parameters or arbitrary functions. This naturally leads to the group classification problem of differential equations. The first group classification problem was carried out by Ovsiannikov [1] who classified all forms of the non-linear heat equation $u_{t}=\left(f(u) u_{x}\right)_{x}$. Studies related to group properties of non-linear wave equations began with the well-known paper of Ames [2] in 1981. Since then the group classification problem has been studied intensely for fundamental equations arising from models in engineering and physics, 
e.g. the symmetry classification of the geodesic equations of Riemannian spaces [34, the symmetry classification of the two and three dimensional Newtonian systems [5/6/7/8 and many others 9 9|10/11|12|13|14|15|16|17|18|19|20|21|22|23|24|25]. Furthermore, a symmetry analysis of wave equation in a power-law Bianchi III spacetime spacetime can be found in [26] and a symmetry analysis of the wave equation on static spherically symmetric spacetimes, with higher symmetries, was recently carried out in 27

In 28, it was proved that for a linear, in the derivatives, second order partial differential equation (PDE) the Lie point symmetries are related with the conformal algebra of the geometry defined by the PDE.

One important equation which belongs to this type of PDEs is the Klein Gordon equation

$$
\Delta u+V\left(x^{i}\right) u=0
$$

where $\Delta=\frac{1}{\sqrt{|g|}} \frac{\partial}{\partial x^{2}}\left(\sqrt{|g|} \frac{\partial}{\partial x^{3}}\right)$ is the Laplace operator defined in terms of the metric of the Riemannian space.

In [29] the generic Lie symmetry vector for the Klein Gordon equation in a Riemannian space has been found and it has been expressed in terms of the elements of the conformal algebra of the space modulo a constraint relation involving the Lie symmetry vector and the potential entering the Klein Gordon equation.

Concerning the Noether point symmetries of the Klein Gordon equation in a Riemannian space we prove that these are the same as the Lie point symmetries of this equation.

These geometric results transfer the problem of the Lie / Noether symmetry classification of the Klein Gordon equation (1) in a Riemannian space to the problem of determining the CKVs of the space and the appropriate potentials which solve the corresponding constraint condition. We note that taking the potential $V\left(x^{i}\right)=0$ this classification provides, as a special case, the corresponding symmetry analysis for the wave equation in Bianchi I spacetimes.

These results can be useful in two ways. Using the reduction of the Klein Gordon equation by means of certain Lie point symmetries one determines invariant solutions of (11) with respect to these symmetries. Furthermore because the Lie symmetries are also Noether symmetries one is able to determine conserved currents for the corresponding potential.

We apply these general results in Bianchi I spacetime and perform the complete group classification of the Lie / Noether point symmetries of Klein Gordon equation in this spacetime in terms of the potential $V\left(x^{i}\right)$.

The paper is organized as follows. Section 2 provides the geometrical preliminaries and the theoretical background. In section 3 we give the explicit form of the generic Lie symmetry vector of the Klein Gordon equation in a general Riemannian space in terms of the conformal algebra of the space modulo a constraint condition involving the potential. In section 4 we obtain the symmetry classification for the 
Klein Gordon equation in Bianchi I spacetimes and the corresponding potentials. Furthermore, in section 5 the Lie point symmetries are applied in order to reduce the Klein Gordon equation and determine invariant solutions. Finally in section 6 we draw our conclusion and we discuss the symmetry analysis of the wave equation.

\section{Preliminaries}

In this section we give the basic definitions and properties of the collineations of spacetime and of the point symmetries of differential equations.

\subsection{Collineations of Riemannian spaces}

A collineation in a Riemannian space is a vector field $\mathbf{X}$ which satisfies an equation of the form

$$
\mathcal{L}_{X} \mathbf{A}=\mathbf{B}
$$

where $\mathcal{L}_{X}$ is the Lie derivative with respect to the vector field $\mathbf{X}, \mathbf{A}$ is a geometric object (not necessarily a tensor) defined in terms of the metric and its derivatives and $\mathbf{B}$ is an arbitrary tensor with the same indices as the geometric object $\mathbf{A}$. The collineations of Riemannian spaces have been classified by Katzin et.al. 34. In the following we are interested in the collineations of the metric tensor i.e. $\mathbf{A}=g_{i j}$.

If there exists a function $\psi\left(x^{k}\right)$ so that $\mathbf{B}=2 \psi\left(x^{k}\right) g_{i j}$ the vector field $X$ is called a Conformal Killing vector (CKV) if $\psi\left(x^{k}\right) \neq 0$, a special CKV (sp.CKV) if $\psi_{; a b}=0$, a homothetic (HV) if $\psi=$ constant and a Killing vector (KV) if $\psi=0$.

The CKVs of the metric $g_{i j}$ form a closed Lie algebra which is called the conformal algebra of the metric $g_{i j}$. Two metrics $g_{i j}, \bar{g}_{i j}$ which are conformally related, that is there exist a function $N^{2}\left(x^{k}\right)$ such as $\bar{g}_{i j}=N^{2}\left(x^{k}\right) g_{i j}$, have the same conformal algebra. A space is called conformally flat if it is conformally related to the flat space,

The conformal algebra contains two closed subalgebras, the Homothetic algebra and the Killing algebra related as follows

$$
K V s \subseteq H V s \subseteq C K V s .
$$

The dimension of the conformal algebra of a $n$-dimensional metric $(n>2)$ of constant curvature is $\frac{1}{2}(n+1)(n+2)$, the dimension of the Killing algebra is $\frac{1}{2} n(n+1)$ and the dimension of the Homothetic algebra is $\frac{1}{2} n(n+1)+1$. The flat space admits $\frac{1}{2}(n+1)(n+2)$ CKVs.

\subsection{Point symmetries of differential equations}

A partial differential equation (PDE) is a function $H=H\left(x^{i}, u^{A}, u_{, i}^{A}, u_{, i j}^{A}\right)$ in the jet space $\bar{B}_{\bar{M}}$, where $x^{i}$ are the independent variables and $u^{A}$ are the dependent variables. The infinitesimal point transformation

$$
\begin{aligned}
\bar{x}^{i} & =x^{i}+\varepsilon \xi^{i}\left(x^{k}, u^{B}\right), \\
\bar{u}^{A} & =u^{A}+\varepsilon \eta^{A}\left(x^{k}, u^{B}\right),
\end{aligned}
$$


A. Paliathanasis, M. Tsamparlis and M.T. Mustafa

has the infinitesimal symmetry generator

$$
\mathbf{X}=\xi^{i}\left(x^{k}, u^{B}\right) \partial_{x^{i}}+\eta^{A}\left(x^{k}, u^{B}\right) \partial_{u^{A}}
$$

The generator $\mathbf{X}$ of the infinitesimal transformation (2),(3) is called a Lie point symmetry of the PDE $H$ if there exists a function $\lambda$ such that the following condition holds [30]31]

$$
\mathbf{X}^{[n]}(H)=\lambda H, \bmod H=0,
$$

where

$$
\mathbf{X}^{[n]}=\mathbf{X}+\eta_{i}^{A} \partial_{u_{i}^{A}}+\eta_{i j}^{A} \partial_{u_{i j}^{A}}+\ldots+\eta_{i_{1} i_{2} \ldots i_{n}}^{A} \partial_{u_{i_{1} i_{2} \ldots i_{n}}^{A}}
$$

is the $n^{\text {th }}$ prolongation vector and

$$
\eta_{i}^{A}=\eta_{, i}^{A}+u_{, i}^{B} \eta_{, B}^{A}-\xi_{, i}^{j} u_{, j}^{A}-u_{, i}^{A} u_{, j}^{B} \xi_{, B}^{j},
$$

with

$$
\begin{aligned}
\eta_{i j}^{A} & =\eta_{, i j}^{A}+2 \eta_{, B(i}^{A} u_{, j)}^{B}-\xi_{, i j}^{k} u_{, k}^{A}+\eta_{, B C}^{A} u_{, i}^{B} u_{, j}^{C}-2 \xi_{,(i|B|}^{k} u_{j)}^{B} u_{, k}^{A} \\
& -\xi_{, B C}^{k} u_{, i}^{B} u_{, j}^{C} u_{, k}^{A}+\eta_{, B}^{A} u_{, i j}^{B}-2 \xi_{,(j}^{k} u_{, i) k}^{A}-\xi_{, B}^{k}\left(u_{, k}^{A} u_{, i j}^{B}+2 u_{(, j}^{B} u_{, i) k}^{A}\right)
\end{aligned}
$$

Lie point symmetries of differential equations can be used in order to determine invariant solutions or transform solutions to solutions [33. From condition (5) one defines the Lagrange system

$$
\frac{d x^{i}}{\xi^{i}}=\frac{d u}{\eta}=\frac{d u_{i}}{\eta_{[i]}}=\ldots=\frac{d u_{i j . . i_{n}}}{\eta_{\left[i j \ldots i_{n}\right]}}
$$

whose solution provides the characteristic functions

$$
W^{[0]}\left(x^{k}, u\right), W^{[1] i}\left(x^{k}, u, u_{i}\right), \ldots, W^{[n]}\left(x^{k}, u, u, i, \ldots, u_{i j \ldots i_{n}}\right) .
$$

The solution $W^{[k]}$ is called the kth order invariant of the Lie point symmetry vector (4). These invariants can be used in order to reduce the order of the PDE (for details see e.g. [31]).

The Lie point symmetries of a PDE span a Lie algebra $G_{L}$ of dimension $\operatorname{dim} G_{L}>1$. The application of a Lie symmetry to a PDE $H$ leads to a new differential equation $\bar{H}$ which is different from $H$ and is possible to admit Lie symmetries which are not Lie symmetries of $H$ (these Lie symmetries are called Type II hidden symmeties). It has been shown [32] that if $X_{1}, X_{2}$ are Lie point symmetries of the original PDE with commutator $\left[X_{1}, X_{2}\right]=c X_{1}$ where $c$ is a constant, then reduction by $X_{2}$ results in $X_{1}$ being a point symmetry of the reduced PDE $\bar{H}$ while reduction by $X_{1}$ results in a PDE $\bar{H}$ which has no relevance for the PDE $\bar{H}$.

For PDEs arising from a variational principle, Noether's theorem states [33].

Theorem 1. The action of the generator (4) of the infinitesimal transformation (2), (3) on the Lagrangian $L=L\left(x^{k}, u^{A}, u_{k}^{A}\right)$ leaves $H\left(x^{i}, u^{A}, u_{, i}^{A}, u_{, i j}^{A}\right)$ invariant 
if there exists a vector field $A^{i}=A^{i}\left(x^{i}, u^{A}\right)$ such that the following condition is satisfied

$$
\mathbf{X}^{[1]} L+L D_{i} \xi^{i}=D_{i} A^{i} .
$$

The corresponding Noether flow $I^{i}$ is defined by the expression

$$
I^{i}=\xi^{k}\left(u_{k}^{A} \frac{\partial L}{\partial u_{i}^{A}}-\delta_{k}^{i} L\right)-\eta^{A} \frac{\partial L}{\partial u_{i}^{A}}+A^{i} .
$$

and it is conserved, that is satisfies the relation

$$
D_{i} I^{i}=0 .
$$

In (11) $D_{i}$ is the total derivative, defined as follows:

$$
D_{i}=\partial_{x^{i}}+u_{i}^{A} \partial_{u^{A}}+u_{i j}^{A} \partial_{u_{j}^{A}}+\ldots
$$

\section{Point symmetries of the Klein Gordon equation in a general Riemannian space}

In a recent paper 29] it has been shown that the Lie point symmetries of the Klein Gordon equation (11) in a general Riemannian space are elements of the conformal algebra of the space. More specifically the following theorem is proved.

Theorem 2. The Lie point symmetries of the Klein Gordon equation (1) in a Riemannian space of dimension $n$ are generated from the elements of the conformal algebra of the metric $g_{i j}$ defining the Laplace operator, as follows

a) for $n>2$ the Lie symmetry vector is

$$
X=\xi^{i}\left(x^{k}\right) \partial_{i}+\left(\frac{2-n}{2} \psi\left(x^{k}\right) u+a_{0} u+b\left(x^{k}\right)\right) \partial_{u}
$$

where $\xi^{i}$ is a $C K V$ with conformal factor $\psi\left(x^{k}\right), b\left(x^{k}\right)$ is a solution of (11) and the following condition involving the potential is satisfied

$$
\xi^{k} V_{, k}+2 \psi V-\frac{2-n}{2} \Delta \psi=0
$$

b) for $n=2$ the Lie symmetry vector is

$$
X=\xi^{i}\left(x^{k}\right) \partial_{i}+\left(a_{0} u+b\left(x^{k}\right)\right) \partial_{u}
$$

where $\xi^{i}$ is a $C K V$ with conformal factor $\psi\left(x^{k}\right), b\left(x^{k}\right)$ is a solution of (1) and the following condition is satisfied

$$
\xi^{k} V_{, k}+2 \psi V=0 .
$$

The Klein Gordon equation (1) follows from the Lagrangian

$$
L\left(x^{i}, u, u, i\right)=\frac{1}{2} \sqrt{g} g^{i j} u_{, i} u_{, j}-\frac{1}{2} \sqrt{g} V\left(x^{i}\right) u^{2} .
$$


A. Paliathanasis, M. Tsamparlis and M.T. Mustafa

For each term of the Noether symmetry condition (9) we have

$$
\begin{gathered}
D_{i} A^{i}=A_{, i}^{i}+A_{, u}^{i} u_{, i} \\
L D_{i} \xi^{i}=L \xi_{, i}^{i}+L \xi_{, u}^{i} u_{, i} \\
\mathbf{X}^{[1]} L=\xi^{i} \frac{\partial L}{\partial x^{i}}+\eta \frac{\partial L}{\partial u}+\eta_{i} \frac{\partial L}{\partial u_{i}} .
\end{gathered}
$$

Therefore by collecting the terms of the same powers of $\left(u_{i}\right)^{K}$ we find that condition (9) is equivalent to the following determining system of equations

$$
\begin{gathered}
\xi_{, u}^{k}=0, \sqrt{g} g^{i j} \eta_{, j}=A_{, u}^{i} \\
-\frac{1}{2} \sqrt{g}\left(\ln (\sqrt{g})_{, k} \xi^{k} V u^{2}+V_{, k} \xi^{k} u^{2}-2 V u \eta+\xi_{, k}^{k} V u^{2}\right)=A_{, i}^{i} \\
g_{, k}^{i j} \xi^{k}-2 g^{k(i} \xi_{, k}^{j)}+2 g^{i j} \eta_{, u}+g^{i j} \xi_{, k}^{k}+\ln (\sqrt{g})_{, k} \xi^{k} g^{i j}=0 .
\end{gathered}
$$

The solution of this system is as follows. Equation (20) implies $\xi^{i}=\xi^{i}\left(x^{k}\right)$ and $A_{i}=\sqrt{g} \int \eta_{, i} d u+\Phi_{i}\left(x^{k}\right)$. Combining this with $(\sqrt{g})_{, k}=\sqrt{g} \Gamma_{k r}^{r}$ where $\Gamma_{j k}^{i}$ are the connection coefficients, condition (22) becomes

$$
\mathcal{L}_{\xi} g_{i j}=\left(\xi_{; k}^{k}+2 \eta_{, u}\right) g_{i j}
$$

which means that $\xi^{i}=\xi^{i}\left(x^{k}\right)$ is a CKV of the metric $g_{i j}$ and that $\xi_{; k}^{k}=n \psi\left(x^{k}\right)$. However since the Noether point symmetries are also Lie point symmetries, from (12) we have that $\eta\left(x^{k}, u\right)=\frac{2-n}{2} \psi\left(x^{k}\right) u+a_{0} u+b\left(x^{k}\right)$; where $\Delta b+V\left(x^{i}\right) b=0$. Then condition (21) becomes

$$
V_{, k} \xi^{k} u^{2}+2 \psi V u^{2}+a_{0} V u=\frac{2-n}{2} \Delta \psi u^{2}-\frac{2}{\sqrt{g}} \Phi_{, i}^{i} .
$$

This equation is an identity hence the coefficients of the various powers of $u$ must vanish. It follows then that $a_{0}=0, \Phi_{, i}^{i}=0$ and the Noether condition becomes

$$
V_{, k} \xi^{k}+2 \psi V-\frac{2-n}{2} \Delta \psi=0
$$

which is the Lie point symmetry condition (13). We conclude that every Lie point symmetry (but not the trivial vector field $u \partial_{u}$, since $a_{0}=0$ ) of the Klein Gordon equation is also a Noether point symmetry for the Lagrangian (16). Therefore we have the following theorem

Theorem 3. The Noether point symmetries of the Klein Gordon Lagrangian (16) are generated by the CKVs of the metric $g_{i j}$. The generic Noether point symmetry vector is the Lie generic point symmetric vector with $a_{0}=0$ and corresponding gauge function

$$
A_{i}=\frac{2-n}{4} \sqrt{g} \psi_{, i}\left(x^{k}\right) u^{2}+\Phi_{i}\left(x^{k}\right)
$$


where $\Phi_{i}\left(x^{k}\right)$ is a function which satisfies the condition $D_{i} \Phi^{i}=0$.

From Noether's theorem the function $\Phi_{i}\left(x^{k}\right)$ can be absorbed in the Noether flow, hence without loss of generality we can set $\Phi_{i}\left(x^{k}\right)=0$.

Theorem 3 is a generalization of the result of [35] for the Klein Gordon equation with constant potential, i.e. $V\left(x^{k}\right)=V_{0}$. Furthermore, from Theorem 3 we have the following corollary which relates the dimension of the algebras of Lie and Noether point symmetries of the Klein Gordon equation.

Corollary 4. If the Klein Gordon equation (1) with Lagrangian (16) admits $n$ Lie point symmetries which span the Lie algebra $G_{L S}$, $\operatorname{dim} G_{L S}=n$, then the Lagrangian (16) admits as Noether point symmetries the Lie algebra $G_{N S}$, of dimension $\operatorname{dim} G_{N S}=n-1$, where $G_{L S}=G_{N S} \cup\left\{u \partial_{u}\right\}$.

In the following we apply theorems 2 and 3 in order to determine the potentials for which the Klein Gordon equation (11) admits Lie/Noether point symmetries in (diagonal) Bianchi I spacetimes.

\section{The Lie and Noether point symmetries of the Klein Gordon equation in Bianchi I spacetime}

In order to apply the results of theorems 2 and 3 we need the complete conformal algebra of Bianchi I spacetime.

\subsection{The conformal algebra of the diagonal Bianchi I spacetime}

The Bianchi type $N(N=I, \ldots, I X)$ models are spatially homogeneous spacetimes which admit a group of motions $G_{3}$ [36] acting on spacelike hypersurfaces. Some of these spacetimes are non-isotropic generalizations of the Friedman-RobertsonWalker (FRW) space-time such as the Bianchi I, V and IX spacetimes. These spacetimes have been used in the discussion of anisotropies in a primordial universe and its evolution towards the observed isotropy of the present epoch.

The simplest type of these spacetimes are the Bianchi I models which correspond to the abelian group $G_{3}$ consisting of the three $\mathrm{KVs}$

$$
Y_{I}^{1}=\partial_{x}, Y_{I}^{2}=\partial_{y}, Y_{I}^{3}=\partial_{z} .
$$

In synchronous coordinates the metric of this spacetime is

$$
d s^{2}=-d t+A^{2}(t) d x+B^{2}(t) d y+C^{2}(t) d z
$$

where $A(t), B(t), C(t)$ are functions of the time coordinate $t$ only.

When the metric functions $A(t), B(t), C(t)$ satisfy certain relations it is possible that the resulting Bianchi I spacetimes admit a larger conformal algebra. In 37] it has been shown that these spacetimes can be classified in two sets a. The Bianchi I spacetimes which are non-conformally flat (which we refer as Class A) and b. The Bianchi I spacetimes which are conformally flat (which we refer as Class B). In 
A. Paliathanasis, M. Tsamparlis and M.T. Mustafa

more formal terms the first family contains the Petrov type I spacetimes and the second family the Petrov type D spacetimes.

Before we proceed it will be useful if we clarify the following point. From theorem 2 we have that in order the Klein Gordon equation (30) to admits Lie /Noether point symmetries in a Riemannian space two conditions must be satisfied:

a. The metric has to admit a non-void conformal algebra, which is always the case because all Bianchi I metrics admit the three $\mathrm{KVs} Y_{I}^{\mu}$.

b. The potential $V(t, x, y, z)$ must satisfy the constraint condition (13) or (15) depending on the dimension of the space i.e. $n>2$ or $n=2$ respectively.

This means that for each CKV of the conformal algebra of Bianchi I spacetimes given in [37] we must solve condition (13) (because $n=4$ ) and find those potentials for which it is satisfied. In other words the constraint condition (13) acts as a double selection rule selecting for each $\mathrm{CKV}$ a corresponding potential or, if this is not possible, abandoning the CKV for being a Lie point symmetry of the Klein Gordon equation.

\subsection{The general Bianchi I spacetime}

The generic line element of Bianchi I spacetime is (28) with $A(t), B(t), C(t)$ being general smooth functions of $t$ and admits the abelian group of isometries $G_{3}$ consisting of the vector fields $Y_{I}^{1}, Y_{I}^{2}, Y_{I}^{3}$ (see equation (27))

For the line element (28) the Lagrangian (16) becomes

$L\left(x^{i}, u, u_{, i}\right)=\frac{1}{2} A B C\left(-u_{, t}^{2}+A^{-2} u_{, x}^{2}+B^{-2} u_{, y}^{2}+C^{-2} u_{, z}^{2}\right)-\frac{1}{2} A B C V(t, x, y, z) u^{2}$

and the Klein Gordon equation (1) is

$$
-u_{, t t}+A^{-2} u_{, x x}+B^{-2} u_{, y y}+C^{-2} u_{, z z}-\left(\frac{\dot{A}}{A}+\frac{\dot{B}}{B}+\frac{\dot{C}}{C}\right) u_{, t}+V(t, x, y, z) u=0 .
$$

For each of the vectors $Y_{I}^{1-3}$ and their linear combinations we solved condition (13) and found the potentials $V(t, x, y, z)$ of table 1 .

Having considered the general case we continue with the special cases of Class A and Class B of Bianchi I spacetimes.

\subsection{Class A: Bianchi Spacetimes}

In the special case where $B^{2}(t)=C^{2}(t)$ and $A^{2}(t) \neq B^{2}(t)$ the spacetime (28) admits the extra $\mathrm{KV} Y_{I}^{4}=z \partial_{y}-y \partial_{z}$ 远.

The vector field $Y_{I}^{4}$ and all linear combinations with the vector fields $Y_{I}^{1-3}$ when introduced in the condition (13) give as solutions the results of table 2

a Similarly if $A^{2}(t)=B^{2}(t), A^{2}(t) \neq C^{2}(t)$ or $A^{2}(t)=C^{2}(t), A^{2}(t) \neq B^{2}(t)$ the spacetime (28) admits a four dimension Killing algebra where the corresponding extra KVs are $y \partial_{x}-x \partial_{y}$ or $z \partial_{x}-x \partial_{z}$ respectively. These cases being similar we restrict our study to the case where $B^{2}(t)=C^{2}(t)$. 
Table 1. Point symmetries and potentials for the Klein Gordon equation in the Bianchi I spacetime for arbitrary functions $A(t), B(t)$ and $C(t)$

\begin{tabular}{ccc}
\hline \hline Potential & Lie Sym. & Noether Sym. \\
\hline$V(t, x, y, z)$ & $X_{u}=\partial_{u}$ & No \\
$V(t, y, z)$ & $Y_{I}^{1}$ & Yes \\
$V(t, x, z)$ & $Y_{I}^{2}$ & Yes \\
$V(t, x, y)$ & $Y_{I}^{3}$ & Yes \\
$V\left(t, y-\frac{b}{a} x, z\right)$ & $a Y_{I}^{1}+b Y_{I}^{2}$ & Yes \\
$V\left(t, z-\frac{b}{a} x, y\right)$ & $a Y_{I}^{1}+b Y_{I}^{3}$ & Yes \\
$V\left(t, x, z-\frac{b}{a} y\right)$ & $a Y_{I}^{2}+b Y_{I}^{3}$ & Yes \\
$V\left(t, y-\frac{b}{a} x, z-\frac{c}{a} x\right)$ & $a Y_{I}^{1}+b Y_{I}^{2}+c Y_{I}^{3}$ & Yes \\
\hline \hline
\end{tabular}

Table 2. Point symmetries and potentials for the Klein Gordon equation in the Bianchi I spacetime with $A(t) \neq B(t)=C(t)$

\begin{tabular}{ccc}
\hline \hline Potential & Lie Sym. & Noether Sym. \\
\hline$V\left(t, x, y^{2}+z^{2}\right)$ & $Y_{I}^{4}$ & Yes \\
$V\left(t, x-\frac{a}{b} \arctan \frac{y}{z}, y^{2}+z^{2}\right)$ & $a Y_{I}^{1}+b Y_{I}^{4}$ & Yes \\
$V\left(t, x, \frac{1}{2}\left(y^{2}+z^{2}\right)+\frac{a}{b} z\right)$ & $a Y_{I}^{2}+b Y_{I}^{4}$ & Yes \\
$V\left(t, x, \frac{1}{2}\left(y^{2}+z^{2}\right)+\frac{a}{b} y\right)$ & $a Y_{I}^{3}+b Y_{I}^{4}$ & Yes \\
$V\left(t, x-\frac{a}{c} \arctan \frac{c y}{b+c z}, \frac{1}{2}\left(y^{2}+z^{2}\right)+\frac{b}{c} z\right)$ & $a Y_{I}^{1}+b Y_{I}^{2}+c Y_{I}^{4}$ & Yes \\
$V\left(t, x-\frac{a}{c} \arctan \frac{c y-b}{c z}, \frac{1}{2}\left(y^{2}+z^{2}\right)+\frac{b}{c} y\right)$ & $a Y_{I}^{1}+b Y_{I}^{3}+c Y_{I}^{4}$ & Yes \\
$V\left(t, x, \frac{c}{2}\left(y^{2}+z^{2}\right)-(b y-a z)\right)$ & $a Y_{I}^{2}+b Y_{I}^{3}+c Y_{I}^{4}$ & Yes \\
$V\left(t, x-\frac{a}{d} \arctan \left(\frac{d y-c}{d z+c}\right), \frac{d}{2}\left(y^{2}+z^{2}\right)-(c y-b z)\right)$ & $a Y_{I}^{1}+b Y_{I}^{2}+c Y_{I}^{3}+d Y_{I}^{4}$ & Yes \\
\hline \hline
\end{tabular}

When the functions $A(t), B(t)$ and $C(t)$ satisfy the relations

$$
A(t)=\frac{1}{U(t)} \bar{A}(t), B(t)=\frac{1}{U(t)} \bar{B}(t), C(t)=\frac{1}{U(t)} \bar{C}(t)
$$

where

$$
\bar{A}(t)=e^{-\alpha \int U(t) d t}, \bar{B}(t)=e^{-\beta \int U(t) d t}, \bar{C}(t)=e^{-\gamma \int U(t) d t}
$$

then the Bianchi I spacetime admits the proper CKV

$$
Y_{I}^{5}=\frac{1}{U(t)} \partial_{t}+\alpha x \partial_{x}+\beta y \partial_{y}+\gamma z \partial_{z}
$$

with conformal factor $\psi_{I}^{5}=-\frac{\dot{U}}{U^{2}}$ 37. We study two cases (a) the conformal factor $\psi_{I}^{5}$ is a solution of Laplace equation $\Delta \psi_{I}^{5}=0$ and (b) the conformal factor $\psi_{I}^{5}$ is not a solution of Laplace equation. 
Table 3. Point symmetries and potentials for the Klein Gordon equation in the Bianchi I spacetime with $A(t), B(t), C(t)$ are given from (31) and $U(t)$ from (34)

\begin{tabular}{ccc}
\hline \hline Potential $V(t, x, y, z)=U^{2}(t) \bar{V}(t, x, y, z)$ & Lie Sym. & Noether Sym. \\
\hline $\bar{V}(x \bar{A}, y \bar{B}, z \bar{C})$ & $Y_{I}^{5}-\psi_{I}^{5} u \partial_{u}$ & Yes \\
$\bar{V}\left(\left(x+\frac{1}{\alpha} \frac{a}{b}\right) \bar{A}, y \bar{B}, z \bar{C}\right)$ & $a Y_{I}^{1}+b Y_{I}^{5}-b \psi_{I}^{5} u \partial_{u}$ & Yes \\
$\bar{V}\left(x \bar{A},\left(y+\frac{1}{\beta} \frac{a}{b}\right) y \bar{B}, z \bar{C}\right)$ & $a Y_{I}^{2}+b Y_{I}^{5}-b \psi_{I}^{5} u \partial_{u}$ & Yes \\
$\bar{V}\left(x \bar{A}, y \bar{B},\left(z+\frac{1}{\gamma} \frac{a}{b}\right) \bar{C}\right)$ & $a Y_{I}^{3}+b Y_{I}^{5}-b \psi_{I}^{5} u \partial_{u}$ & Yes \\
$\bar{V}\left(\left(x+\frac{1}{\alpha} \frac{a}{c}\right) \bar{A},\left(y+\frac{1}{\beta} \frac{b}{c}\right) \bar{B}, z \bar{C}\right)$ & $a Y_{I}^{1}+b Y_{I}^{2}+c Y_{I}^{5}-c \psi_{I}^{5} u \partial_{u}$ & Yes \\
$\bar{V}\left(\left(x+\frac{1}{\alpha} \frac{a}{c}\right) \bar{A}, y \bar{B},\left(z+\frac{1}{\gamma} \frac{b}{c}\right) \bar{C}\right)$ & $a Y_{I}^{1}+b Y_{I}^{3}+c Y_{I}^{5}-c \psi_{I}^{5} u \partial_{u}$ & Yes \\
$\bar{V}\left(x \bar{A},\left(y+\frac{1}{b} \frac{a}{c}\right) \bar{B},\left(z+\frac{1}{\gamma} \frac{b}{c}\right) \bar{C}\right)$ & $a Y_{I}^{2}+b Y_{I}^{3}+c Y_{I}^{5}-c \psi_{I}^{5} u \partial_{u}$ & Yes \\
$\bar{V}\left(\left(x+\frac{1}{\alpha} \frac{a}{d}\right) \bar{A},\left(y+\frac{1}{\beta} \frac{b}{d}\right) \bar{B},\left(z+\frac{1}{\gamma} \frac{c}{d}\right) \bar{C}\right)$ & $a Y_{I}^{1}+b Y_{I}^{2}+c Y_{I}^{3}+d Y_{I}^{5}-d \psi_{I}^{5} u \partial_{u}$ & Yes \\
\hline \hline
\end{tabular}

In case (a), the conformal factor $\psi_{I}^{5}$ is a solution of the Laplace equation $\Delta \psi_{I}^{5}=0$ if the function $U(t)$ satisfies the condition

$$
L^{(3)}+\left(3 L^{(1)}-\alpha-\beta-\gamma\right) \frac{L^{(2)}}{L}=0, \text { where } L=\frac{1}{U(t)} .
$$

In order to find an exact solution of equation (33) we use the Lie symmetry method. By applying the Lie symmetry condition (5) where the generator of the infinitesimal transformation is $\mathbf{X}_{L}=\xi(t, L) \partial_{t}+\eta(t, L) \partial_{L}$, we find that equation (33) admits the extra Lie point symmetries $\partial_{t}, t \partial_{t}+L \partial_{L}$. Therefore from the application of invariants of Lie point symmetries in equation (33), we find the solutions

$$
L_{1}(t)=\frac{1}{M} W\left(\exp \left(M^{2}(\alpha, \beta, \gamma)\left(t+c_{1}\right)\right)\right)+\frac{1}{M(\alpha, \beta, \gamma)}, L_{2}(t)=t
$$

where $M(\alpha, \beta, \gamma)=\alpha+\beta+\gamma$ and $W(t)$ is the Lambert $\mathrm{W}$-function.

We observe that for the solution $L_{2}(t)$ of (33), the conformal factor $\psi_{I}^{5}$ becomes a constant; that is, the $\mathrm{CKV} Y_{I}^{5}$ reduces to a HV. This is the only Bianchi I spacetime which admits a proper HV. In table 3 we give the potentials which admit a Lie / Noether point symmetry which is generated by the vector field $Y_{I}^{5}$ and all linear combinations of the vector field $Y_{I}^{5}$ with the $\mathrm{KVs} Y_{I}^{1-3}$.

In case (b), where $U(t)$ is arbitrary, the Klein Gordon equation (30) admits the Lie/ Noether point symmetries of table 3 when

$$
V(t, x, y, z)=U^{2} \bar{V}(t, x, y, z)-\frac{1}{U} U^{(2)}+\left(\frac{3 U^{(1)}}{U^{2}}+M(\alpha, \beta, \gamma)\right) U^{(1)} .
$$

and $\bar{V}(t, x, y, z)$ are taken from table 3 .

Furthermore when $\bar{B}(t)=\bar{C}(t)$, i.e. $\beta=\gamma$ in (32), the spacetime (28) admits also the additional $\mathrm{KV} Y_{I}^{4}$. Then the general (extra) point symmetry vector is $X_{G I}=\sum_{\alpha=1}^{4} a_{\alpha} Y_{I}^{\alpha}+a_{5} X_{I}^{5}$ where $X_{I}^{5}=Y_{I}^{5}-\psi_{I}^{5} u \partial_{u}$. In table 4 we give the 
Table 4. Commutators of the elements of the generic Lie symmetry vector of the Klein Gordon equation in a Bianchi I spacetime where $A(t), B(t)=C(t)$ are given from (31) and $U(t)$ from (34)

\begin{tabular}{c|ccccc}
\hline \hline$[.,]$. & $Y_{I}^{1}$ & $Y_{I}^{2}$ & $Y_{I}^{3}$ & $Y_{I}^{4}$ & $X_{I}^{5}$ \\
\hline$Y_{I}^{1}$ & 0 & 0 & 0 & 0 & $\alpha Y_{I}^{1}$ \\
$Y_{I}^{2}$ & & 0 & 0 & $-Y_{I}^{3}$ & $\beta Y_{I}^{2}$ \\
$Y_{I}^{3}$ & & & 0 & $Y_{I}^{2}$ & $\beta Y_{I}^{3}$ \\
$Y_{I}^{4}$ & & & & 0 & 0 \\
$X_{I}^{5}$ & & & & & 0 \\
\hline \hline
\end{tabular}

commutators of the elements of the generic point symmetry $X_{G I}$. These will be used in the reduction of the Klein Gordon equation.

\subsection{Class B: Bianchi I spacetime is conformally flat}

According to 37] the line element of the conformally flat Bianchi I spacetime (28) (excluding the FRW spacetime) is of the following two forms

$$
d s^{2}=-d t^{2}+\sin ^{2} t d x^{2}+\cos ^{2} t d y^{2}+d z^{2}
$$

and

$$
d s^{2}=-d t^{2}+\sinh ^{2} t d x^{2}+\cosh ^{2} t d y^{2}+d z^{2} .
$$

The conformal algebra of these spaces consists of 15 vector fields, in which the seven vector fields are KVs and the remaining eight vector fields are proper CKVs [37.

The corresponding Lagrangian of the Klein Gordon equation in each space is:

$$
L_{1}=\frac{\sin 2 t}{4}\left(-u_{, t}^{2}+u_{, z}^{2}+(\sin t)^{-2} 1 u_{, x}+(\cos t)^{-2} u_{, y}^{2}\right)-\frac{\sin 2 t}{4} V(t, x, y, z) u^{2}
$$

and

$$
L_{2}=\frac{\sinh 2 t}{4}\left(-u_{, t}^{2}+u_{, z}^{2}+(\sinh t)^{-2} u_{, x}+(\cosh t)^{-2} u_{, y}^{2}\right)-\frac{\sinh 2 t}{4} V(t, x, y, z) u^{2}
$$

The Euler-Lagrange equation of (38) is the Klein Gordon equation

$$
-u_{, t t}+\sin ^{-2} t u_{, x x}+\cos ^{-2} t u_{, y y}+u_{, z z}+(\tan t-\cot t) u_{, t}+V(t, x, y, z) u=0
$$

and the Euler-Lagrange equation of (39) gives

$$
-u_{, t t}+\sinh ^{-2} t u_{, x x}+\cosh ^{-2} t u_{, y y}+u_{, z z}-(\tanh t+\operatorname{coth} t) u_{, t}+V(t, x, y, z) u=0
$$

The Klein Gordon equations (40) and (41) are related by the complex coordinate transformation $(t, x, y, z) \rightarrow(i \bar{t}, i \bar{x}, i \bar{y}, i \bar{z})$, therefore in the following we study only the Lie point symmetries of equation (40). 
Table 5. Point symmetries and potentials for the Klein Gordon equation in the Bianchi I spacetime with metric (36)

\begin{tabular}{ccc}
\hline \hline Potential & Lie Sym. & Noether Sym. \\
\hline$V(x+\ln (\sin t), y+\ln (\cos t), z)$ & $\bar{Y}_{I}^{4}$ & Yes \\
$V(x+\ln (\sin t), y-\ln (\cos t), z)$ & $\bar{Y}_{I}^{5}$ & Yes \\
$V(x-\ln (\sin t), y+\ln (\cos t), z)$ & $\bar{Y}_{I}^{6}$ & Yes \\
$V(x-\ln (\sin t), y-\ln (\cos t), z)$ & $\bar{Y}_{I}^{7}$ & Yes \\
\hline \hline
\end{tabular}

For the metric (36) the seven KVs are the three vector fields $Y_{I}^{1-3}$ and the four vector fields

$$
\begin{gathered}
\bar{Y}_{I}^{4}=e^{x+y}\left(\partial_{t}-\cot t \partial_{x}+\tan t \partial_{y}\right), \bar{Y}_{I}^{5}=e^{x-y}\left(\partial_{t}-\cot t \partial_{x}-\tan t \partial_{y}\right) \\
\bar{Y}_{I}^{6}=e^{-x+y}\left(\partial_{t}+\cot t \partial_{x}+\tan t \partial_{y}\right), \bar{Y}^{7}=e^{-x-y}\left(\partial_{t}+\cot t \partial_{x}-\tan t \partial_{y}\right) .
\end{gathered}
$$

Concerning the eight CKVs and their corresponding conformal factors these are:

$$
\begin{aligned}
& C_{ \pm x}^{1}=e^{ \pm x}\left(\cos t \cos z \partial_{t} \mp \frac{\cos z}{\sin t} \partial_{x}-\sin t \sin z \partial_{z}\right), \psi_{ \pm x}^{1}=-e^{ \pm x} \sin t \cos z \\
& C_{ \pm x}^{2}=e^{ \pm x}\left(\cos t \sin z \partial_{t} \mp \frac{\sin z}{\sin t} \partial_{x}+\sin t \cos z \partial_{z}\right), \psi_{ \pm x}^{2}=-e^{ \pm x} \sin t \sin z \\
& C_{ \pm y}^{1}=e^{ \pm y}\left(\sin t \sin z \partial_{t} \pm \frac{\sin z}{\cos t} \partial_{y}-\cos t \cos z \partial_{z}\right), \psi_{ \pm y}^{1}=e^{ \pm y} \cos t \sin z \\
& C_{ \pm y}^{2}=e^{ \pm y}\left(\sin t \cos z \partial_{t} \pm \frac{\cos z}{\cos t} \partial_{y}+\cos t \sin z \partial_{z}\right), \psi_{ \pm y}^{2}=e^{ \pm y} \cos t \cos z
\end{aligned}
$$

Having the complete conformal algebra of the space we continue with the application of theorems 2 and 3 in order to determine the potentials for which the Klein Gordon equation (40) admits Lie and Noether point symmetries. For convenience and economy of space the results are presented in tabular form. In table 5 we give the form of the potentials for which the Lie and the Noether point symmetries are generated by the Killing subalgebra $\left\{\bar{Y}^{4-7}\right\}$ of the metric (36) and in table 6 we give the potentials for the point symmetries which are generated by the proper Conformal algebra $\left\{C_{ \pm(x, y)}^{1-2}\right\}$ of the metric (36).

\section{Lie invariant solutions of the Klein Gordon equation in Bianchi I spacetimes}

In this section we apply the zero order invariants of some Lie point symmetries of the Klein Gordon equation (30) in order to reduce the differential equation and determine invariant solutions with respect to these symmetries. We will study the reduction for the two class $\mathrm{A}$ and $\mathrm{B}$ of section 4 
Table 6. Point symmetries and potentials for the Klein Gordon equation in the Bianchi I spacetime with metric (36)

\begin{tabular}{ccc}
\hline \hline Potential & Lie Sym. & Noether Sym. \\
\hline $1-\frac{1}{2 \cos ^{2} t}+\frac{1}{\cos ^{2} t} V\left(x \pm \ln \left(\frac{1-\cos 2 t}{\sin 2 t}\right), y, \frac{\cos t}{\sin z}\right)$ & $C_{ \pm x}^{1}-\psi_{ \pm x}^{1} u \partial_{u}$ & Yes \\
$1-\frac{1}{2 \cos ^{2} t}+\frac{1}{\cos ^{2} t} V\left(x \pm \ln \left(\frac{1-\cos 2 t}{\sin 2 t}\right), y, \frac{\cos t}{\cos z}\right)$ & $C_{ \pm x}^{2}-\psi_{ \pm x}^{2} u \partial_{u}$ & Yes \\
$1-\frac{1}{2 \sin ^{2} t}+\frac{1}{\sin ^{2} t} V\left(x, y \mp\left(\frac{1-\cos 2 t}{\sin 2 t}\right), \frac{\sin t}{\cos z}\right)$ & $C_{ \pm y}^{1}-\psi_{ \pm y}^{1} u \partial_{u}$ & Yes \\
$1-\frac{1}{2 \sin ^{2} t}+\frac{1}{\sin ^{2} t} V\left(x, y \mp\left(\frac{1-\cos 2 t}{\sin 2 t}\right), \frac{\sin t}{\sin z}\right)$ & $C_{ \pm y}^{2}-\psi_{ \pm y}^{2} u \partial_{u}$ & Yes \\
\hline \hline
\end{tabular}

\subsection{Class A: Invariant solutions}

For the class A Bianchi I spacetimes we will study the reduction for the of the Klein Gordon eqation for two cases, (I) when $V(t, x, y, z)=V(t)$ and $A^{2}(t) \neq$ $B^{2}(t) \neq C^{2}(t)$ and (II) when $V(t, x, y, z)=V\left(\left(y^{2}+z^{2}\right) e^{-2 \beta \int U(t) d t}\right) U^{2}(t)$ where $A(t), B(t), C(t)$ are given from (31) and $U(t)=\frac{1}{t}$.

Case I: When $V=V(t)$ from table 1 the Klein Gordon equation (30) admits as extra Lie point symmetries the vector fields $Y_{I}^{1-3}$. However in order to apply the Lie invariants we choose the symmetries

$$
X_{I}^{\alpha}=Y_{I}^{\alpha}+\mu_{\alpha} X_{u}, \alpha=1,2,3
$$

which are linear combinations with the symmetry $X_{u}$. Furthermore, for the commutators we have $\left[X_{I}^{a}, X_{I}^{\beta}\right]=0$, where $\alpha, \beta=1,2,3$. The zero order invariants of $X_{I}^{1}$ are $\left\{t, y, z, e^{-\mu_{1} x} u\right\}$. We select $\{t, y, z\}$ to be the independent variables and $u(t, x, y, z)=e^{\mu_{1} x} v(t, y, z)$, where $v(t, y, z)$ is the dependent variable. By replacing in (30) we find the reduced equation

$$
-v_{, t t}+B^{-2} v_{, y y}+C^{-2} v_{, z z}-\left(\frac{\dot{A}}{A}+\frac{\dot{B}}{B}+\frac{\dot{C}}{C}\right) v_{, t}+\left(V(t)+\mu_{1}^{2}\right) v=0 .
$$

The Lie point symmetries $X_{I}^{2,3}$ are inherited symmetries of (42); therefore, by applying the zero order invariants the solution of the Klein Gordon equation (30) is

$$
u(t, x, y, z)=\exp \left(\mu_{1} x+\mu_{2} y+\mu_{3} z\right) w(t)
$$

where $w(t)$ is the solution of the second order ODE

$$
\ddot{w}+\left(\frac{\dot{A}}{A}+\frac{\dot{B}}{B}+\frac{\dot{C}}{C}\right) \dot{w}-\left(V(t)+\mu_{1}^{2}+\mu_{2}^{2}+\mu_{3}^{2}\right) w=0 .
$$

This is the damped oscillator which admits eight Lie point symmetries therefore can be transformed to the equation $W^{\prime \prime}+\omega^{2} W=0$ by an appropriate transformation $[38$. 
Case II: In the second case we consider the potential $V(t, x, y, z)=\frac{1}{t^{2}} V^{\prime}\left(\frac{y^{2}+z^{2}}{t^{\beta}}\right)$ and we select the line element of the Bianchi I spacetime to be

$$
d s^{2}=-d t^{2}+t^{2-2 \alpha} d x^{2}+t^{2-2 \beta}\left(d y^{2}+d z^{2}\right)
$$

hence the corresponding Klein Gordon equation is

$$
-u_{, t t}+t^{2 \alpha-2} u_{, x x}+t^{2 \beta-2}\left(u_{, y y}+u_{, z z}\right)-\frac{3-\alpha-2 \beta}{t} u_{, t}+\frac{1}{t^{2}} V^{\prime}\left(\frac{y^{2}+z^{2}}{t^{2 \beta}}\right) u=0
$$

From tables 1, 2] and 3, we have that the Klein Gordon equation (46) admits three extra Lie point symmetries, the $Y_{I}^{1}, Y_{I}^{4}$ and the $X_{I}^{5}=Y_{I}^{5}-u \partial_{u}$, where $\psi_{I}^{5}=1$ since for the metric (45) $Y_{I}^{5}$ is a HV. In order to apply the zero order invariants we select the symmetries

$$
X_{I}^{1}=Y_{I}^{1}+\mu_{1} X_{u}, X_{I}^{4}=Y_{I}^{4}+\mu_{2} X_{u}, X_{I}^{5^{\prime}}=X_{I}^{5}+\mu_{5} X_{u}
$$

with commutators

$$
\left[X_{I}^{1}, Y_{I}^{4}\right]=0,\left[X_{I}^{5^{\prime}}, X_{I}^{4}\right]=0,\left[X_{I}^{1}, X_{I}^{5^{\prime}}\right]=\alpha Y_{I}^{1} .
$$

We start the reduction with the vector field $X_{I}^{4}$. The zero order invariants are $\left\{t, x, y^{2}+z^{2}, e^{-\mu_{4} \arctan \frac{z}{y}} u\right\}$. We select $t, x$ and $r=x^{2}+y^{2}$ to be the independent variables and $u(t, x, y, z)=e^{\mu_{4} \theta} v(t, x, r)$, where $v(t, x, r)$ is the dependent variable and $\theta=\arctan \frac{z}{y}$. Replacing in (46) we find the reduced equation

$$
-v_{, t t}+t^{2 \alpha-2} v_{, x x}+t^{2 \beta-2}\left(v_{, r r}+\frac{1}{r} v_{, r}\right)-\frac{3-\alpha-2 \beta}{t} v_{, t}+\frac{1}{t^{2}} \bar{V}\left(\frac{r^{2}}{t^{2 \beta}}\right) v=0
$$

where $\bar{V}\left(\frac{r^{2}}{t^{2 \beta}}\right)=V^{\prime}\left(\frac{r^{2}}{t^{2 \beta}}\right)+\mu_{4} \frac{t^{2 \beta}}{r^{2}}$. Equation (48) admits as Lie point symmetries the vector fields $X_{I}^{1}, X_{I}^{5^{\prime}}$ which are inherited symmetries. We continue with the application of the zero order invariants of $X_{I}^{1}$. These are $\left\{t, r, e^{-\mu_{1} x} v\right\}$, hence we select as independent variables the $t, r$ and as dependent variable the $w(t, r)=$ $e^{-\mu_{1} x} v$. By replacing in (48) we have

$$
-w_{, t t}+t^{2 \beta-2}\left(w_{, r r}+\frac{1}{r} w_{, r}\right)-\frac{3-\alpha-2 \beta}{t} w_{, t}+\frac{1}{t^{2}}\left(\bar{V}\left(\frac{r^{2}}{t^{2 \beta}}\right)+\mu_{1}^{2} t^{2 \alpha}\right) w=0
$$

However, from the commutator (47) the Lie point symmetry $X_{I}^{5^{\prime}}$ is an inherited symmetry when $X_{I}^{1}=Y_{I}^{1}$, i.e. $\mu_{1}=0$, or when $\alpha=0$. From the vector field $X_{I}^{5^{\prime}}$ we have that the zero order invariants are $\zeta=r t^{-\beta}$ and $w(t, r)=t^{\mu_{5}} \sigma(\zeta)$. Therefore the reduced equation is

$$
\left(1-\zeta^{2} \beta^{2}\right) \sigma_{, \zeta \zeta}-\beta \zeta\left(3 \beta-2-2 \mu_{5}-\frac{1}{\beta \zeta}\right) \sigma_{, \zeta}+K(\zeta) \sigma=0 .
$$

When $\mu_{1}=0, K(\zeta)=2 \beta \mu_{5}+\bar{V}(\zeta)$ and the solution of the Klein Gordon equation (46) is

$$
u(t, x, y, z)=t^{\mu_{5}} \exp \left(\mu_{4} \arctan \left(\frac{z}{y}\right)\right) \sigma\left(\sqrt{y^{2}+z^{2}} t^{-\beta}\right)
$$


Table 7. Commutators of the elements of the generic Lie point symmetry vector of the Klein Gordon equation in a Bianchi I spacetime with metric (36)

\begin{tabular}{c|ccccccc}
\hline \hline$[.,]$. & $Y_{I}^{1}$ & $Y_{I}^{2}$ & $Y_{I}^{3}$ & $\bar{Y}_{I}^{4}$ & $\bar{Y}_{I}^{5}$ & $\bar{Y}_{I}^{6}$ & $\bar{Y}_{I}^{7}$ \\
\hline$Y_{I}^{1}$ & 0 & 0 & 0 & $\bar{Y}_{I}^{4}$ & $\bar{Y}_{I}^{5}$ & $-\bar{Y}_{I}^{6}$ & $-\bar{Y}_{I}^{7}$ \\
$Y_{I}^{2}$ & & 0 & 0 & $\bar{Y}_{I}^{4}$ & $-\bar{Y}_{I}^{5}$ & $\bar{Y}_{I}^{6}$ & $-\bar{Y}_{I}^{7}$ \\
$Y_{I}^{3}$ & & & 0 & 0 & 0 & 0 & 0 \\
$\bar{Y}_{I}^{4}$ & & & & 0 & 0 & 0 & $-4\left(Y_{I}^{1}+Y_{I}^{2}\right)$ \\
$\bar{Y}_{I}^{5}$ & & & & & 0 & $4\left(-Y_{I}^{1}+Y_{I}^{2}\right)$ & 0 \\
$\bar{Y}_{I}^{6}$ & & & & & & 0 & 0 \\
$\bar{Y}_{I}^{7}$ & & & & & & & 0 \\
\hline \hline
\end{tabular}

When $\alpha=0, K(\zeta)=2 \beta \mu_{5}+\mu_{1}^{2}+\bar{V}(\zeta)$ and the solution of the Klein Gordon equation (46) is

$$
u(t, x, y, z)=t^{\mu_{5}} \exp \left(\mu_{1} x+\mu_{4} \arctan \left(\frac{z}{y}\right)\right) \sigma\left(\sqrt{y^{2}+z^{2}} t^{-\beta}\right)
$$

where $\sigma\left(\sqrt{y^{2}+z^{2}} t^{-\beta}\right)=\sigma(\zeta)$ satisfies the second order ODE (50).

\subsection{Class B: Invariant solutions}

In this section, we use the Lie symmetries of the Klein Gordon equation (40) in order to find invariant analytic solutions. We shall do that for the point symmetries generated by the KVs only. We shall consider only the potentials $V_{ \pm x}=V(x \pm \ln (\sin t))$ and $V_{ \pm y}=V(y \pm \ln (\cos t))$.

For the reduction of (40) we need the commutators of the KVs of the metric (36) which are given in table 7.

Potential $V_{+x}=\boldsymbol{V}(\boldsymbol{x}+\ln (\sin t))$ From tables 1 and $[5$ we read that for this potential the Klein Gordon equation (40) admits four extra Lie point symmetries given by the vector fields $Y_{I}^{2}, Y_{I}^{3}, \bar{Y}_{I}^{4}, \bar{Y}_{I}^{5}$.

In order to reduce equation (40) and determine an invariant solution we need a double reduction of the equation, therefore we must have a Lie point symmetry of the reduced equation. This is assured if we use Lie point symmetries which commute hence reduction by any of them inherits the remaining vector to the reduced equation. In our case we shall use for reduction the Lie point symmetries

$$
X_{I}^{3}=Y_{I}^{3}+\mu_{3} X_{u}, \quad \bar{X}_{I}^{4}=\bar{Y}_{I}^{4}+\mu_{4} X_{u}, \bar{X}_{I}^{5}=\bar{Y}_{I}^{5}+\mu_{5} X_{u}
$$

of table 1 because from table 7 we have that all their commutators vanish.

Reduction by $X_{I}^{3}$ leads to the reduced equation

$-v_{, t t}+\sin ^{-2} t v_{, x x}+\cos ^{-2} t v_{, y y}+(\tan t-\cot t) v_{, t}+\left(V(x+\ln (\sin t))+\mu_{3}^{2}\right) v=0$ 
where $u(t, x, y, z)=v(t, x, y) e^{\mu_{3} z}$. As we explained above, equation (53) inherits the vector fields $\bar{X}_{I}^{4}, \bar{X}_{I}^{5}$ as Lie point symmetries. Reduction of (153) by $\bar{X}_{I}^{4}, \bar{X}_{I}^{5}$ leads to the solution of the Klein Gordon equation:

$$
u(t, x, y, z)=\sigma(\zeta) \exp \left(\mu_{3} z-\frac{\cot t}{2}\left(\mu_{4} e^{-(x+y)}+\mu_{5} e^{x-y}\right)\right.
$$

where $\zeta=x+\ln (\sin t)$ and $\sigma(\zeta)$ satisfies the second order ODE

$$
\sigma_{, \zeta \zeta}+2 \sigma_{, \zeta}+\left(V(\zeta)+\mu_{3}^{2}-\mu_{4} \mu_{5} e^{-2 \zeta}\right) \sigma=0 .
$$

In the case where $V(\zeta)=e^{-2 \zeta} \mu_{4} \mu_{5}$, i.e.

$$
V(x+\ln (\sin t))=\mu_{4} \mu_{5} \exp (-(x+\ln (\sin t)))
$$

the solution of (55) is

$$
\sigma(\zeta)=\sigma_{1} \exp \left(\left[-1+\sqrt{1-\mu_{3}^{2}}\right] \zeta\right)+\sigma_{2} \exp \left(\left[-1-\sqrt{1-\mu_{3}^{2}}\right] \zeta\right) .
$$

Potential $\boldsymbol{V}_{-\boldsymbol{x}}=\boldsymbol{V}(\boldsymbol{x}-\ln (\sin t))$ When $V(t, x, y, z)=V(x-\ln (\sin t))$, the Klein Gordon equation (40) admits the extra Lie point symmetries $Y_{I}^{2}, Y_{I}^{3}, \bar{Y}_{I}^{6}, \bar{Y}_{I}^{7}$. With the same reasoning as in the previous case we use for the reduction the KVs

$$
X_{I}^{3}=Y_{I}^{3}+\mu_{3} X_{u}, \quad \bar{X}_{I}^{6}=\bar{Y}_{I}^{6}+\mu_{6} X_{u}, \bar{X}_{I}^{7}=\bar{Y}_{I}^{7}+\mu_{7} X_{u}
$$

which span an Abelian algebra. Successive reduction of (40) by $X_{I}^{3}, \bar{X}_{I}^{6}$ and $\bar{X}_{I}^{7}$ results in the solution

$$
u(t, x, y, z)=\sigma(\xi) \exp \left(\mu_{3} z-\frac{\cot t}{2}\left(\mu_{6} e^{x-y}+\mu_{7} e^{x+y}\right)\right) .
$$

where $\xi=x-\ln (\sin t)$ and $\sigma(\xi)$ is a solution of the equation:

$$
\sigma_{, \xi \xi}-2 \sigma_{, \xi}+\left(V(\zeta)+\mu_{3}^{2}-\mu_{6} \mu_{7} e^{2 \xi}\right) \sigma=0
$$

Again we note that for the potential $V_{-x}=\mu_{6} \mu_{7} \exp (2 \xi)-\mu_{3}^{2}$ the solution (57) of the Klein Gordon equation (40) is:

$u(t, x, y, z)=\left[\sigma_{0}+\sigma_{1} \exp \left(2 x-\ln \left(\sin ^{2} t\right)\right)\right] \exp \left(\mu_{3} z-\frac{\cot t}{2}\left(\mu_{6} e^{x-y}+\mu_{7} e^{x+y}\right)\right)$

Potentials $\boldsymbol{V}_{ \pm \boldsymbol{y}}$ : When $V(t, x, y, z)=V(y+\ln (\cos t))$, the Klein Gordon equation (40) admits the extra Lie point symmetries $Y_{I}^{1}, Y_{I}^{3}, \bar{Y}_{I}^{4}, \bar{Y}_{I}^{6}$. For the reduction in this case we select the vectors $X_{I}^{3}, \bar{X}_{I}^{4}, \bar{X}_{I}^{6}$ and find the solution:

$$
u(t, x, y, z)=\sigma(\zeta) \exp \left(\mu_{3} z+\frac{\tan t}{2}\left(\mu_{4} e^{-x-y}+\mu_{6} e^{x-y}\right)\right)
$$

where $\zeta=y+\ln (\cos t)$ and $\sigma(\zeta)$ satisfies equation (55) with $\mu_{5}=\mu_{6}$. 
When $V(t, x, y, z)=V(y-\ln (\cos t))$, the Klein Gordon equation (40) admits the extra Lie point symmetries $Y_{I}^{1}, Y_{I}^{3}, \bar{Y}_{I}^{5}, \bar{Y}_{I}^{7}$. In this case, we reduce equation (40) with the Lie algebra $\left\{X_{I}^{3}, \bar{X}_{I}^{5}, \bar{X}_{I}^{7}\right\}$ and we find the following invariant solution

$$
u(t, x, y, z)=\sigma(\xi) \exp \left(\mu_{3} z+\frac{\tan t}{2}\left(\mu_{5} e^{-x+y}+\mu_{7} e^{x+y}\right)\right)
$$

where $\xi=y-\ln (\cos t)$ and $\sigma(\xi)$ satisfies equation (58) with $\mu_{6}=\mu_{5}$.

\section{Conclusion}

The knowledge of the Lie point symmetries of a differential equation is important because it can be used to determine invariant solutions of the equation. In this work we considered the Klein Gordon (1) in a general Riemannian space and proved that (a) The Lie point symmetries of (1) coincide with the Noether point symmetries of Lagrangian (16) (b) The generators of the Lie/ Noether point symmetries are the CKVs and their linear combinations (c) Not all CKVs of the space are Lie / Noether point symmetries of (11); A CKV is a Lie / Noether point symmetry of (1) if the constraint condition (13) has a solution for the corresponding potential.

This general results transfer the problem of determining the Lie and the Noether point symmetries of the Klein Gordon equation in a general Riemannian space to the problem of determining the CKVs of the space and the solution of an easy differential condition.

We have applied the general results in the case of the Bianchi I spacetime. The complete conformal algebra of the Bianchi I spacetimes has been determined in 37 . We have used the results of [37] in order a. To determine all potentials for which the resulting Klein Gordon equation in Bianchi I spacetime admits Lie and Noether point symmetries and b. To determined the Lie /Noether symmetry vectors. Due to the plethora of cases and for easy reference the results are presented in the form of tables. The usefulness of these tables is that they provide the appropriate Lie symmetries which can be used for the reduction of the Klein Gordon equation in Bianchi I spacetimes and subsequently the determination of corresponding invariant solutions.

One important byproduct of this study concerns the wave equation. Indeed the latter is obtained from the Klein Gordon equation if one considers the potential to be zero. In this case the constraint condition becomes $\Delta \psi=0$, that is the conformal factor of the CKVs must be a solution of the wave equation This means that the point symmetries of wave equation in Bianchi I spacetimes are the point symmetries of tables 1, 2, 3 and 5, with potential $V(t, x, y, z)=0$ whereas the vector fields of table 6 are not Lie /Noether point symmetries of the wave equation because the conformal factor of the CKVs is not a solution of the wave equation.

Concerning the reduction procedures which we considered in section 4 they remain valid for the wave equation. An exact solution of the wave equation in Kasner universe has been found recently in [39. We would like to note that the 
ansantz of 39 it is based on the group invariants of the wave equation (16) as given in section 5.1 .

A study of the Klein Gordon equation and the symmetries of classical particles in other Bianchi type spacetimes is under investigation and the results will be presented in a subsequent paper.

\section{Acknowledgments}

The authors thank the referee for the useful remarks which improved this work. AP acknowledge financial support of INFN.

\section{References}

[1] L. V. Ovsiannikov, Group analysis of differential equations, Academic Press, New York, (1982).

[2] W. F. Ames, R. J. Lohner and E. Adams, Group properties of $u_{t t}=\left(f(u) u_{x}\right)_{x}$, Int. J. Non-Linear Mech. 16, 439 (1981).

[3] M. Tsamparlis and A. Paliathanasis, Lie symmetries of geodesic equations and projective collineations, Nonlinear Dynamic, 62, (2010), 203

[4] U. Camci, Symmetries of geodesic motion in Gödel-type spacetimes, JCAP 07 (2014) 002

[5] P.A. Damianou and C. Sophocleous, Symmetries of Hamiltonian systems with two degrees of freedom, J. Math. Phys. 40 (1999) 210

[6] P.A. Damianou and C. Sophocleous, Classification of Noether Symmetries for Lagrangians with Three Degrees of Freedom, Nonlinear Dynamics 36 (2004) 3

[7] M. Tsamparlis and A. Paliathanasis, Two-dimensional dynamical systems which admit Lie and Noether symmetries, J.Phys.A: Math. Theor, 44, (2011), 175202, (arXiv:1101.5771)

[8] M. Tsamparlis, A. Paliathanasis and L. Karpathopoulos, Autonomous threedimensional Newtonian systems which admit Lie and Noether point symmetries, J. Phys. A: Math. Theor. 45 (2012) 275201, (arXiv:1111.0810)

[9] N.H. Ibragimov, M. Torrisi and A. Valenti, Preliminary group classification of equations $v_{t t}=f\left(x, v_{x}\right) v_{x x}+g\left(x, v_{x}\right)$, J. Math. Phys., 32, 2988 (1991).

[10] M. Torrisi and R. Tracina, Equivalence transformations and symmetries for a heat conduction model, Internat. J. Non-Linear Mech. 33, 473 (1998).

[11] R.Z. Zhdanov and V.I. Lahno, Group classification of heat conductivity equations with a nonlinear source, J. Phys. A: Math. Gen. 32, 7405 (1999).

[12] M.L. Gandarias, M. Torrisi and A. Valenti, Symmetry classification and optimal systems of a non-linear wave equation, Int. J. Non-Linear Mech. 39, 389 (2004).

[13] R.O. Popovych, N.M. Ivanova, New results on group classification of nonlinear diffusion convection equations, J. Phys. A: Math. Gen. 37, 7547 (2004).

[14] V. Lahno, R. Zhdanov and O. Magda, Group classification and exact solutions of nonlinear wave equations, Acta Appl. Math. 91, 253 (2006).

[15] N.M. Ivanova and C. Sophocleous, On the group classification of variable coefficient nonlinear diffusion convection equations, J. Comput. Appl. Math. 197, 322 (2006).

[16] D. Huang and N.M. Ivanova, Group analysis and exact solutions of a class of variable coefficient nonlinear telegraph equations, J. Math. Phys., 48, 073507 (2007).

[17] F.M. Mahomed. Symmetry group classification of ordinary differential equations: Survey of some results, Math. Methods Appl. Sci. 30, 1995 (2007). 
[18] O.O. Vaneeva, R.O. Popovych and C. Sophocleous, Enhanced group analysis and exact solutions of variable coefficient semilinear diffusion equations with a power source, Acta Appl. Math. 106, 1 (2008).

[19] N.M. Ivanova, C. Sophocleous and P.G.L. Leach, Group classification of a class of equations arising in financial mathematics, J. Math. Anal. Appl. 372, 273 (2010).

[20] H. Azad and M.T. Mustafa, Group classification, optimal system and optimal reductions of a class of Klein Gordon equations, Commun. Nonlinear. Sci. Numer. Simul. 15, 1132 (2010).

[21] R. Tracina, N.M. Ivanova and C. Sophocleous, Group Classification of ThreeDimensional Variable-Coefficient Burgers Equation, Waves and Stability in Continuous Media, 1, 224 (2010).

[22] M. Tsamparlis, A. Paliathanasis and A. Qadir, Noether symmetries and isometries of the minimal surface Lagrangian under constant volume in a Riemannian space, Int. J. Geom. Methods Mod. Phys. 12 (2005) 155003

[23] T. Christodoulakis, N. Dimakis and P.A. Terzis, Lie point and variational symmetries in minisuperspace Einstein gravity, J. Phys. A: Math. Theor., 47, 095202 (2014)

[24] S. Jamal, A. H. Kara and R. Narain, Wave equations in Bianchi Space-times, J. App. Math. 2012, 765361 (2012)

[25] U. Camci, S. Jamal, A.H. Kara, Invariances and Conservation Laws Based on Some FRW Universes, Int J. Theor. Phys., vol.53, 1483 (2014)

[26] S. Jamal, A. H. Kara and A. H. Bokhari,Symmetries, conservation laws, reductions, and exact solutions for the Klein-Gordon equation in de Sitter spacetimes, Can. J. Phys. 90, 667 (2012)

[27] H. Azad, Ahmad Y. Al-Dweik, R. Ghanam and M. T. Mustafa, Symmetry analysis of wave equation on static spherically symmetric spacetimes with higher symmetries, J. Math. Phys., 54, 063509 (2013).

[28] A. Paliathanasis and M. Tsamparlis, Lie point symmetries of a general class of PDEs: The heat equation, J. Geom. Phys., 62 (2012) 2443

[29] A. Paliathanasis and M. Tsamparlis, The geometric origin of Lie point symmetries of the Schrödinger and the Klein-Gordon equations, Int. J. Geom. Methods Mod. Phys. 11 (2014) 1450037

[30] N.H. Ibragimov, Transformation Groups Applied to Mathematical Physics, D. Reidel Publishing Co, Dordrecht (1985).

[31] H. Stephani, "Differential Equations: Their Solutions Using Symmetry", Cambridge University Press, New York, (1989).

[32] K.S. Govinger, Lie subalgebras, reduction of order, and group-invariant solutions, J. Math. Anal. Appl. 258 (2001) 720.

[33] G.W. Bluman and S. Kumei, Symmetries of Differential Equations, (Springer-Verlag, New York, (1989))

[34] G.H. Katzin, J. Levine and R.W. Davis, Curvature Collineations: A Fundamental Symmetry Property of the Space-Times of General Relativity Defined by the Vanishing Lie Derivative of the Riemann Curvature Tensor, J. Math. Phys. 10, 617 (1969)

[35] Y. Bozhkov and I.L. Freire, Special conformal groups of a Riemannian manifold and Lie point symmetries of the nonlinear Poisson equation, J. Differential Equations 249 (2010) 872

[36] M.P.Jr. Rayan and L.C. Shepley, Homogeneous Relativistic Cosmologies, Princeton University Press, Princeton (1975)

[37] M. Tsamparlis and P.S. Apostolopoulos, Symmetries of Bianchi I space-times, J. Math. Phys., 41 (2000) 7573

[38] K.S. Govinder and P.G.L. Leach, An elementary demostration of the existence of 
A. Paliathanasis, M. Tsamparlis and M.T. Mustafa

sl(3,R) symmetry for all second-order linear ordinary differential equations, SIAM Review 40 (1998) 945

[39] E. Battista, E. Di Grezia and G. Esposito, Scalar wave equation in Kasner spacetime, arXiv:1410.3971 